\title{
Ubc9 promotes invasion and metastasis of lung cancer cells
}

\author{
HUI LI, HUIYAN NIU, YANG PENG, JIAHE WANG and PING HE \\ Department of Geriatrics, Shengjing Hospital of China Medical University, Shenyang, Liaoning 110004, P.R. China
}

Received October 29, 2012; Accepted January 7, 2013

DOI: $10.3892 /$ or.2013.2268

\begin{abstract}
Lung cancer is the leading cause of cancer-related mortality worldwide. The mortality is high mainly due to the lack of known effective screening procedures; there is a high tendency for early spread and systemic therapies do not cure metastatic disease. Thus, it is important to investigate the molecular mechanism(s) of lung cancer development and, specifically, to identify an effective method by which to inhibit the invasion and metastasis of lung cancer. Ubiquitinconjugating enzyme 9 (Ubc9), the sole conjugating enzyme for sumoylation, regulates protein function and plays a key role in tumorigenesis. Whether Ubc9 is involved in the invasion and metastasis of lung cancer remains unknown. Herein, we report that Ubc9 exhibits an important role in lung cancer invasion and metastasis. We first investigated the biological effect of Ubc9 on lung cancer by cloning the Ubc9 gene into a eukaryotic expression plasmid and stably expressing it in the human small cell lung cancer cell line NCI-H446 in order to observe any biological changes. We further analyzed the effect of Ubc9 in an in vivo experiment, injecting NCI-H446 cells stably overexpressing Ubc9 into nude mice and analyzing their metastatic ability. Our results demonstrated that Ubc9 is expressed at higher levels in primary lung cancer tissue and metastatic nodules as compared to premalignant and/or normal tissue. Furthermore, we demonstrated that upregulation of Ubc9 expression promotes migration and invasion. Ubc9 likely plays an important role in cancer progression by promoting invasion and metastasis in lung cancer.
\end{abstract}

\section{Introduction}

Despite the significant progress toward the understanding of human lung cancer tumorigenesis in the past two decades, lung cancer remains the leading cause of cancer-related mortality worldwide $(1,2)$. It is estimated that lung and bronchus cancer account for only $12-14 \%$ of new cancer cases per

Correspondence to: Dr Ping He, Department of Geriatrics, Shengjing Hospital of China Medical University, 36 Sanhao Street, Heping, Shenyang, Liaoning 110004, P.R. China

E-mail: hep@sj-hospital.org

Key words: ubiquitin-conjugating enzyme 9, lung cancer, invasion, metastasis, in vivo, cancer progression year, but its early metastatic spread results in a 5-year patient survival of only $10-15 \%$ (3-5). Tumor invasion and metastasis predict poor prognosis in lung cancer and are the main causes of treatment failure and cancer mortality (6). Therefore, it is critical to investigate the molecular mechanism of lung cancer and to find an effective method by which to inhibit its ability to invade and metastasize.

Increasing evidence demonstrates that sumoylation is a multistep process analogous to the ubiquitin pathway and involves maturation, activation, conjugation, ligation, and deconjugation steps. Sumoylation, which includes small ubiquitin-like modifier (SUMO) protein addition or removal from other proteins, is a post-translational modification that is significantly involved in diverse cellular processes, including transcriptional regulation, nuclear transport, cell cycle control, and maintenance of genome integrity through modulating protein-protein interactions of target proteins (7-9). Ubiquitinconjugating enzyme 9 (Ubc9), the sole E2 conjugating enzyme for sumoylation, plays an important role in tumorigenesis and progression (10), such as progression through M phase, DNA damage repair, maintenance of nuclear integrity, and chromosome segregation; in addition, several signaling molecules are targets of Ubc9-mediated sumoylation (11-15). Ubc9 has been reported to be expressed at high levels in advanced melanomas and was found to shield melanoma cells from apoptosis. Blocking Ubc9 expression sensitized melanoma cells to the cytotoxic effects of chemotherapeutic drugs (16). Ubc9 was also reported to be expressed at higher levels in head and neck tumor and lung tumor specimens than in the matched normal tissues (17). However, little is known about the role of Ubc9 in lung cancer and, in particular, about its role in cell invasion and tumor metastasis.

In the present study, we report higher levels of Ubc9 expression in primary lung cancer and metastatic nodules than in premalignant and/or normal tissues. Furthermore, we observed that inducing upregulation of Ubc9 expression in lung cancer cells promotes migration and invasion. Based on these findings, we concluded that Ubc9 may play an important role in cancer progression and that Ubc9 promotes invasion and metastasis in lung cancer.

\section{Materials and methods}

Cell lines and reagents. The lung cancer cell lines A549, NCI-H460, NCI-H446, NCI-H292 were obtained from the American Type Culture Collection. The MCF-7 cell line was a gift from the Department of Cell Biology, China Medical 
University. Cell culture reagents were obtained from Gibco BRL (Grand Island, NY, USA). Protease inhibitors (leupeptin, aprotinin, $\mathrm{Na}_{3} \mathrm{VO}_{4}$, and phenylmethylsulfonylfluoride) were purchased from Sigma Aldrich (St. Louis, MO, USA). Goat antihuman Ubc9 antibody and GAPDH antibody were purchased from Santa Cruz Biotechnology, Inc. (Santa Cruz, CA, USA). Horseradish peroxidase (HRP)-conjugated secondary antibody was obtained from Santa Cruz Biotechnology, Inc. Reagents for SDS-PAGE were obtained from Bio-Rad (Hercules, CA, USA). Chemiluminescent developing reagents and a Micro BCA Protein Assay Reagent kit were obtained from Pierce Chemical Co. (Rockford, IL, USA). All other reagents were of analytical grades.

Patients and tissue samples. Tissue samples were obtained from a total of 143 patients with lung cancer who were treated at Liaoning Cancer Hospital and Institute from 2006 to 2010. Institutional Review Board approval was obtained to procure and analyze the tissues used in this study. None of the patients had received preoperative neo-adjuvant chemotherapy or radiation therapy. Histological types included squamous carcinoma, adenocarcinoma and small cell lung cancer (SCLC) (Table I). No other previous or concomitant primary cancer was present. The surgical specimens were fixed in formalin and embedded in paraffin before they were archived. Ubc9 expression in the tumor specimens was detected using immunohistochemical staining.

Immunohistochemistry. All specimens for immunohistochemical staining were fixed in $10 \%$ neutral formalin, embedded in paraffin and cut in $5-\mu \mathrm{m}$ serial sections. Immunohistochemical staining was performed using a peroxidase-labeled streptavidin-biotin technique. Briefly, tissue sections were deparaffinized and rehydrated. Then, sections were heated in a microwave oven for $10 \mathrm{~min}$ to retrieve antigenicity and were treated with $3 \% \mathrm{H}_{2} \mathrm{O}_{2}$ in methanol for $10 \mathrm{~min}$ to quench endogenous peroxidase activity. After washing in $10 \mathrm{mM}$ PBS (pH 7.6), sections were incubated with $10 \%$ normal goat serum (Solarbio Science and Technology, Beijing, China) for 10 min to block nonspecific antibody binding. Sections were then incubated overnight at $4^{\circ} \mathrm{C}$ with goat antihuman Ubc9 polyclonal antibody (1:100). After washing in PBS, sections were treated with a 1:100 dilution of biotinylated donkey anti-goat IgG for $30 \mathrm{~min}$ followed by a streptavidinperoxidase conjugate for $30 \mathrm{~min}$ (Dako). A solution of $0.02 \%$ diaminobenzidine hydrochloride (DAB) containing $0.03 \%$ $\mathrm{H}_{2} \mathrm{O}_{2}$ was used as chromogen to visualize peroxidase activity. The preparations were lightly counterstained with hematoxylin, mounted with Permount (Thermo Fisher Scientific, Waltham, MA, USA) and examined by light microscopy.

Plasmid construction and cell transfection. Human Ubc9 cDNA (GenBank accession no. NM_003345) was obtained by reverse transcription from $\mathrm{NCI}-\mathrm{H} 446$ cells using the following primers: 5'-CGGAATTCCCACCATGTCGGGGAT-3' and 5'-CGGGATCCAATGAGGGCGCAAAC-3'. PCR conditions were: $95^{\circ} \mathrm{C}$ for $2 \mathrm{~min}\left(94^{\circ} \mathrm{C} 10 \mathrm{sec}, 58^{\circ} \mathrm{C} 1 \mathrm{~min}, 72^{\circ} \mathrm{C} 2.5 \mathrm{~min}\right)$ 30 cycles at $72^{\circ} \mathrm{C}$ for $2 \mathrm{~min}$. The PCR product was cloned into the pGEM-T vector (Promega, Madison, WI, USA). The resulting plasmid, pGEM-T-Ubc9, was sequence-verified. The
Ubc9 fragment was then sub-cloned into the pEGFP-vector (Invitrogen, Carlsbad, CA, USA) using the XhoI and BamHI restriction enzymes. The resulting pEGFP recombinant plasmid containing Ubc9 was also sequence-verified.

Cell culture and transfection. The human lung cancer cells A549, NCI-H460, NCI-H446, NCI-H292 and MCF-7 were maintained in RPMI-1640 medium containing 10\% FBS, $100 \mathrm{IU} / \mathrm{ml}$ penicillin and $100 \mu \mathrm{g} / \mathrm{ml}$ streptomycin. All cell lines were incubated in $5 \% \mathrm{CO}_{2}$ at $37^{\circ} \mathrm{C}$. Stable transfections of NCI-H446 cells were carried out with the following expression vectors: pEGFP-N1-vector and pEGFP-N1-Ubc9. Single cell clones were selected using G418 and confirmed by western blotting.

Western blotting. Western blotting was performed using standard techniques as previously described. Briefly, cells were washed twice with PBS buffer and lysed in RIPA lysis buffer (50 mM Tris- $\mathrm{Cl} \mathrm{pH} \mathrm{7.4,} 150 \mathrm{mM} \mathrm{NaCl}, 0.5 \%$ sodium deoxycholate, $1 \%$ NP-40, 0.1\% SDS, 1 mM EDTA, $100 \mathrm{mM} \mathrm{NaF}, 1 \mathrm{mM}$ $\mathrm{Na}_{3} \mathrm{VO}_{4}, 1 \mathrm{mM}$ PMSF, and $2 \mu \mathrm{g} / \mathrm{ml}$ aprotinin) on ice. Total proteins $(50 \mu \mathrm{g})$ were subjected to sodium dodecyl sulfate-polyacrylamide gel electrophoresis (SDS-PAGE) and transferred to polyvinylidene difluoride membranes. Membranes were blocked with $5 \%$ nonfat milk in TBST (10 mM Tris, $\mathrm{pH} 7.4$, $150 \mathrm{mM} \mathrm{NaCl}$ and $0.1 \%$ Tween-20) at room temperature for $2 \mathrm{~h}$ and incubated with the indicated primary antibodies at $4^{\circ} \mathrm{C}$ overnight with gentle rocking. After washing with TBST, the membrane was reacted with the appropriate HRP-conjugated secondary antibodies for $1 \mathrm{~h}$ at room temperature. After extensive washing with TBST, proteins were visualized by the enhanced chemiluminescence (ECL) detection kit in accordance with the manufacturer's recommendations.

Matrigel in vitro invasion assay. NCI-H446 cells $\left(1.50 \times 10^{4}\right)$ were placed in the upper chamber of $8 \mu \mathrm{m}$ Transwells (Costar Inc.) on $100 \mu \mathrm{l}$ of solid, growth factor reduced Matrigel (BD Biosciences, Bedford, MA, USA). RPMI-1640 medium supplemented with $20 \%$ FBS was added to the wells and then cells were incubated for $4 \mathrm{~h}$ at $37^{\circ} \mathrm{C}$. At the end of the migration assay, the filter side of the upper chamber was cleansed with a cotton swab and the filter was stained for $1 \mathrm{~h}$ with crystal violet (Sigma) in 2\% ethanol and rinsed with water. The filter was gently cut from the chamber and the cells that had migrated through the filter pores from the underside of the filter were counted. Four high-power fields per insert were counted and the values were averaged. Both for NCI-H446+pEGFPN1-vector and NCI-H446+pEGFP-N1-Ubc9, three identical replicates were performed.

Cell scratch migration assay. Cells were seeded in 6-well plates and cultured until confluent. A pipette tip was used to make a straight scratch across the diameter of the dish, simulating a wound. Cell migration was documented using phase contrast microscopy at x10 at 12, 24, 48 and $72 \mathrm{~h}$.

In vivo metastasis assay. Nude mice were anesthetized by intraperitoneal injection of Nembutal [ $45 \mathrm{mg} / \mathrm{kg}$ of pentobarbital $(50 \mathrm{mg} / \mathrm{ml}) /$ saline/ethanol/propylene glycol 10/63/7/18]. The anterior chest wall was scrubbed with $70 \%$ alcohol. A 
Table I. Correlation between Ubc9 expression and clinical pathological factors.

\begin{tabular}{|c|c|c|c|c|}
\hline \multirow[t]{2}{*}{ Variable } & \multirow[t]{2}{*}{ Case (n and \%) } & \multicolumn{2}{|c|}{ The level of Ubc9 expression } & \multirow[t]{2}{*}{$\chi^{2}$ value $(\mathrm{P})$} \\
\hline & & $>$ median values $(\mathrm{n})$ & < median values $(\mathrm{n})$ & \\
\hline \multicolumn{5}{|l|}{ Gender } \\
\hline Male & $97(67.83 \%)$ & 51 & 46 & \multirow[t]{2}{*}{$2.26(>0.05)$} \\
\hline Female & $46(32.17 \%)$ & 18 & 28 & \\
\hline \multicolumn{5}{|l|}{ Age (years) } \\
\hline$>60$ & $51(35.66 \%)$ & 40 & 11 & \multirow[t]{2}{*}{$25.01(<0.01)$} \\
\hline$\leq 60$ & $92(64.34 \%)$ & 32 & 60 & \\
\hline \multicolumn{5}{|l|}{ Histological type } \\
\hline Squamous carcinoma & $31(21.68 \%)$ & 18 & 13 & \multirow[t]{6}{*}{$7.02(>0.05)$} \\
\hline Adenocarcinoma & $28(19.58 \%)$ & 9 & 19 & \\
\hline SCLC & $79(55.25 \%)$ & 42 & 37 & \\
\hline Adenosquamous ca. & $1(0.7 \%)$ & 1 & 0 & \\
\hline Large cell carcinoma & $1(0.7 \%)$ & 0 & 1 & \\
\hline Alveolar carcinoma & $3(1.09 \%)$ & 1 & 2 & \\
\hline \multicolumn{5}{|l|}{ Grade of differentiation } \\
\hline Poor & $95(66.43 \%)$ & 50 & 45 & \multirow[t]{3}{*}{$0.45(>0.05)$} \\
\hline Moderate & $32(22.38 \%)$ & 16 & 16 & \\
\hline Well & $16(11.19 \%)$ & 7 & 9 & \\
\hline \multicolumn{5}{|l|}{ Nodal status } \\
\hline $\mathrm{N}(+)$ & $118(82.52 \%)$ & 78 & 40 & \multirow[t]{2}{*}{$5.94(<0.05)$} \\
\hline $\mathrm{N}(-)$ & $25(17.48 \%)$ & 10 & 15 & \\
\hline \multicolumn{5}{|l|}{ TNM } \\
\hline I & $41(28.67 \%)$ & 15 & 26 & \multirow[t]{4}{*}{$9.57(<0.05)$} \\
\hline II & $32(20.98 \%)$ & 20 & 12 & \\
\hline III & $50(34.97 \%)$ & 28 & 22 & \\
\hline IV & $20(15.38 \%)$ & 15 & 5 & \\
\hline \multicolumn{5}{|l|}{ Survival } \\
\hline Yes & $91(63.64 \%)$ & 33 & 58 & \multirow[t]{2}{*}{$16.12(<0.01)$} \\
\hline No & $52(36.36 \%)$ & 37 & 15 & \\
\hline
\end{tabular}

30-gauge needle on a tuberculin syringe was inserted into the second intercostal space $3 \mathrm{~mm}$ to the left of the sternum and aimed centrally. The spontaneous and continuous entrance of pulsating blood into the transparent needle hub indicated proper positioning of the needle into the left ventricle of the heart. Tumor cells $\left[10^{6}\right.$ cells in $0.1 \mathrm{ml}$ Hank's balanced salt solution (HBSS)] were injected over a 20-40 sec period.

Statistical analysis. Statistical analysis was performed using the Student's t-test. Data are presented as the means \pm SD and p-values $<0.05$ were considered to indicate statistically significant differences. All calculations were carried out using the SPSS13.0 statistical package.

\section{Results}

Expression of Ubc9 in lung cancer patients and its clinical significance. We observed Ubc9 immunostaining in all human lung tissue sections and normal tissue. The Ubc9 protein level was markedly elevated in the lung tumor specimens compared with the normal lung tissue, including both non-small cell lung cancer (NSCLC) and SCLC tissue types. Moreover, the level of Ubc9 protein in NSCLC was higher than in SCLC (Fig. 1A and B). The relationship between the level of Ubc9 expression and the clinicopathological characteristics of lung cancer are summarized in Table I. In this study, the staining intensity of Ubc9 in tumor tissues was assessed as Ubc9 expression levels and was quantified by the IOD values. The IOD values of Ubc9 for the tumor samples ranged from 1663.75 to 64533.7, with a median value of 16523.9. Patients were divided into low and high expression groups, based on whether Ubc9 levels were above or below the median value. There were significant differences correlation between the levels of Ubc9 expression and age, nodal metastasis status, UICC-TNM classification and the probabilities of cancer-specific survival. However, there were no significant differences between the levels of Ubc9 expression and gender, histological type and grade of differentiation. These data suggested that patients with the high Ubc9 expres- 

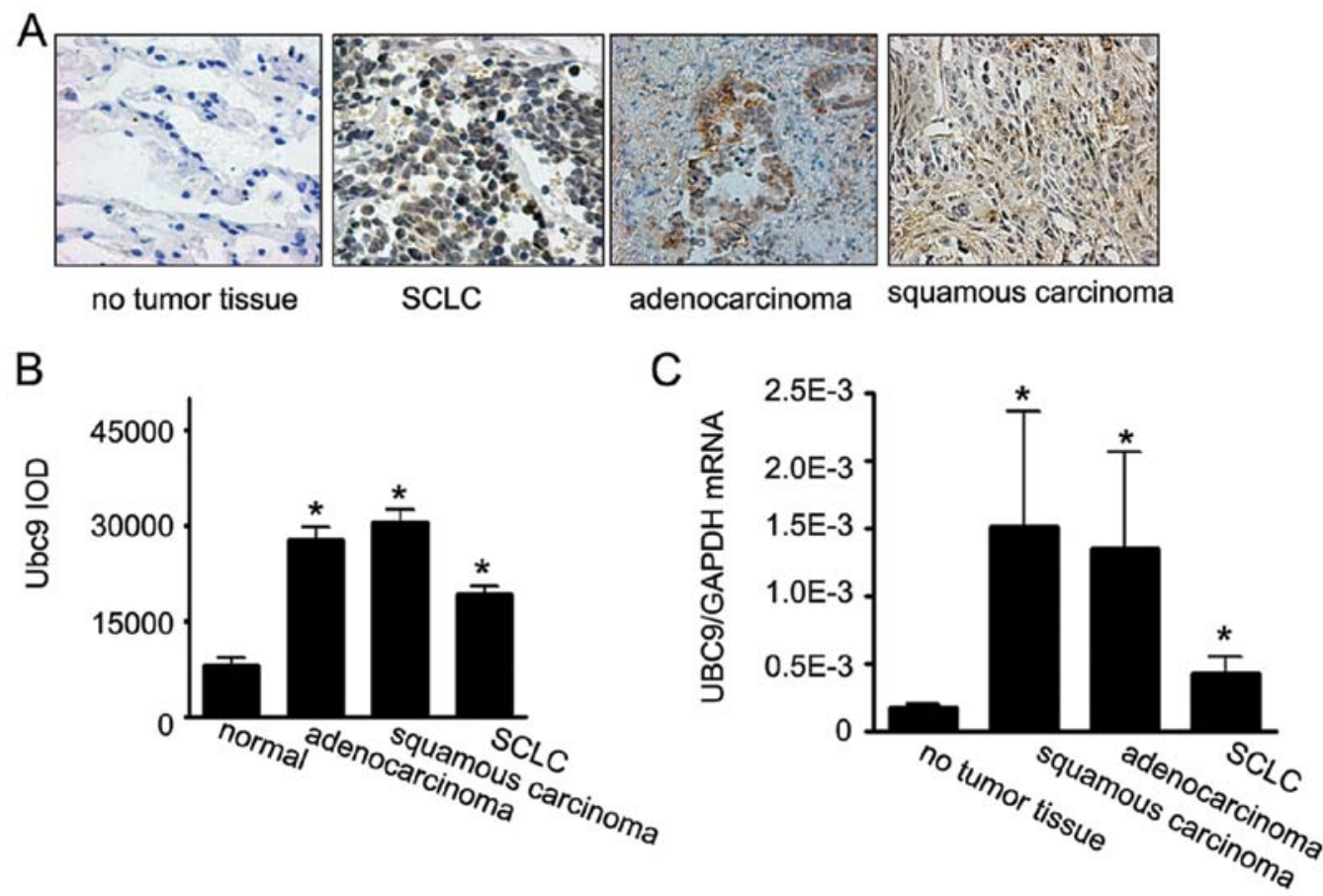

Figure 1. The expression of Ubc9 in lung cancer tissue. (A) The expression of Ubc9 in lung cancer tissues was detected using immunochemistry. (B) The results were analyzed by Image-Pro plus software. (C) The expression of Ubc9 mRNA in lung cancer tissue was detected using real-time PCR. "P<0.05 vs. control.

sion were more likely to have advanced disease than those with the low Ubc9 expression, which correlated with survival of patients with lung cancer.

The level of Ubc9 mRNA in lung cancer tissues was quantitated using real-time PCR. Ubc9 mRNA expression of Ubc9 was again detected in lung normal tissue and lung cancer tissue, including NSCLC and SCLC. Similar to the levels of Ubc9 protein, the level of Ubc9 mRNA in NSCLC samples was significantly higher than in SCLC samples (Fig. 1C). We also tested the level of Ubc9 mRNA and protein using RT-PCR and western blotting in the cell lines, including A549, NCI-H460, NCI-H446, NCI-H292 and MCF-7, and the level of Ubc9 mRNA in NCI-H446 was significantly lower than in other cell lines, as the levels of Ubc9 protein (Fig. 2A and B).

The invasive ability of different lung cancer cells was analyzed using a cell invasion assay (Fig. 2C). The results showed that the ability of lung cancer cells to invade correlated with their levels of Ubc9. These results suggest that Ubc9 promotes lung cancer invasion and metastasis.

Overexpression of Ubc9 enhances NCI-H446 cell invasion and migration. To further investigate the role of Ubc9 in lung cancer, we constructed the mammalian expression vector: pEGFP-N1-Ubc9 and pEGFP-N1-vector, stably transfected NCI-H446 cells, a SCLC cell, generated a stable cell line expressing pEGFP-N1-Ubc9 and pEGFP-N1-vector. Expression of the fusion protein in the pEGFP-N1-Ubc9 clones was detected by western blotting (Fig. 3A). The NCI-H446 cells stably transfected with the pEGFP-N1-vector were used as the control. Transfectants were selected using G418 and the resulting cell lines were designated pEGFP-N1-Ubc9 and pEGFP-N1-vector, respectively. Using a Matrigel cell invasion assay, we found that NCI-H446 cells overexpressing Ubc9 had a
A

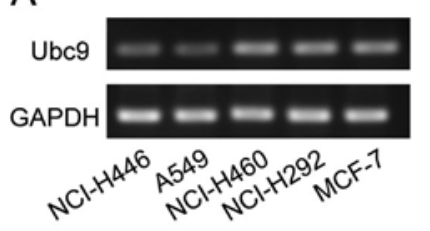

B

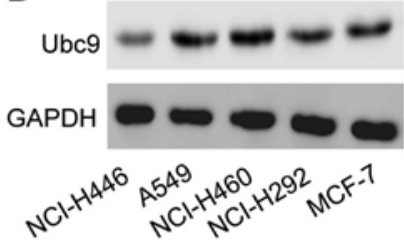

C

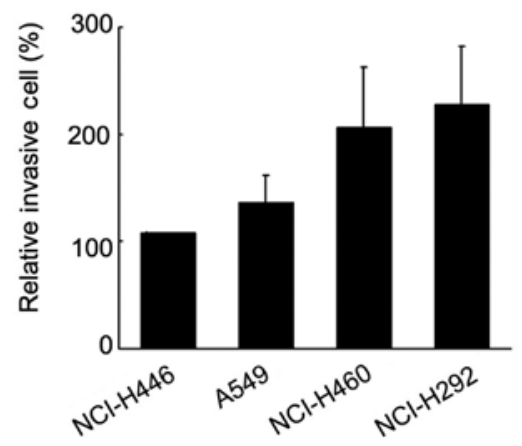

Figure 2. The expression of Ubc9 in lung cancer cells correlates with invasion ability. The expression of Ubc9 in A549, NCI-H460, NCI-H446, NCI-H292 and MCF-7 cells was detected by RT-PCR (A) and western blotting (B). (C) The invasion of lung cancer cells was analyzed by invasion assay. Representative results of three independent experiments are shown. Data represent means \pm SD of three independent experiments.

significantly enhanced ability to invade (Fig. 3B). Furthermore, a cell scratch assay demonstrated that the ability of NCI-H446 cells to migrate was also significantly enhanced (Fig. 3C). These data indicated that higher levels of Ubc9 were sufficient to promote increased invasion and migration in NCI-H446 cells. 
A

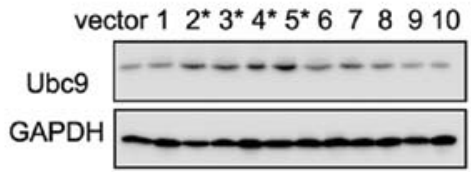

B
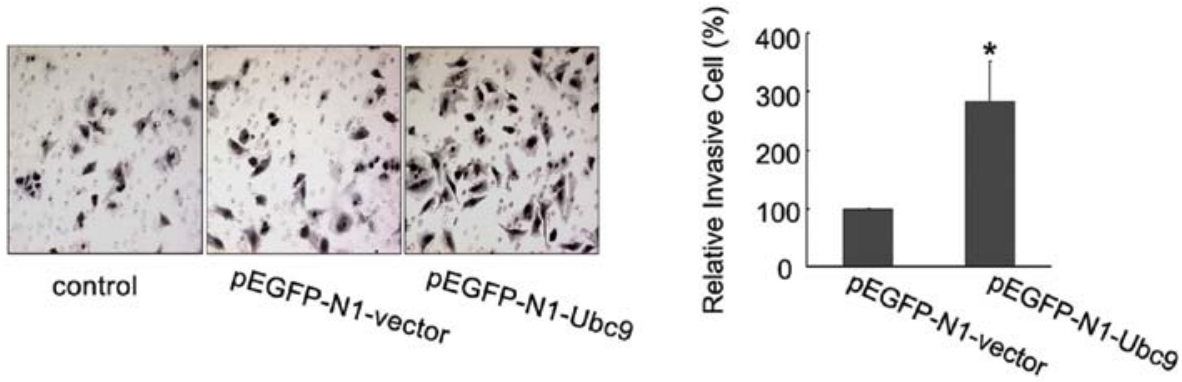

C

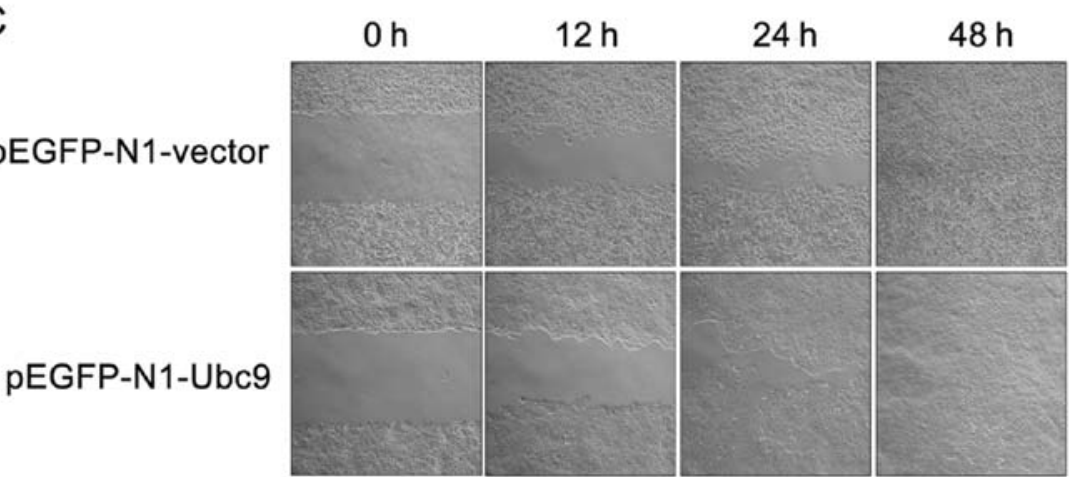

Figure 3. Effect of Ubc9 overexpression in NCI-H446 cells on NCI-H446 cell invasion and migration. (A) NCI-H446 cells transfected with Ubc9 cDNA and pEGFP-N1 vector were designated as pEGFP-N1-Ubc9 and pEGFP-N1-vector, respectively. Ubc9 expression in transfected cells was analyzed by western blotting. (B) Invasion ability was analyzed using a Matrigel invasion assay; migration was analyzed using a cell scratch assay (C). Results representative of three independent experiments are shown. Data is presented as means $\pm \mathrm{SD}$. ${ }^{*} \mathrm{P}<0.05$ vs. control.

Ubc9 promotes NCI-H446 cell lung metastasis in vivo. To directly test the hypothesis that Ubc9 has a causal role in the metastasis of lung cancer cells, we used stable transfection of Ubc9 cDNA to increase the level of Ubc9 protein in NCI-H446 cells. Then, we injected these cells into the left heart ventricle of nude mice in order to evaluate their ability to metastasize to the lung. Injection of either the pEGFP-N1vector/NCI-H446 cells or the pEGFP-N1-Ubc9/NCI-H446 cells produced lung metastatic lesions in the injected mice. Some visible metastatic nodules were observed even in nude mice injected with either the pEGFP-N1 vector/NCI-H446 or the pEGFP-N1-Ubc9/NCI-H446 cells. However, the metastatic ability of pEGFP-N1-Ubc9/NCI-H446 cells was markedly increased compared with that of the control cells (Table II). The pattern of lung metastases in the pEGFPUbc9/NCI-H446 cells was characterized by the formation of multiple foci; however, only one unique metastatic nodule occurred in mice injected with the pEGFP-N1 vector/ NCI-H446 cells (Fig. 4A). The metastatic tumors formed in the lung had morphological characteristics typical of SCLC tumors (Fig. 4B). By contrast, no metastatic nodule formation was observed in the lungs of the mice injected with HBSS. These results show that upregulation of Ubc9 expression enhances lung metastasis of human SCLC cells.
Table II. Effects of upregulation of Ubc9 expression on lung metastasis in nude mice.

\begin{tabular}{|c|c|c|c|}
\hline & \multirow[b]{2}{*}{ Cell line } & \multicolumn{2}{|c|}{ Lung metastasis } \\
\hline & & Incidence & Survival \\
\hline \multirow[t]{3}{*}{ NCI-H446 cells } & HBSS & $0 / 4$ & All >90 \\
\hline & pEGFP-N1-vector & $3 / 10$ & 56 to $>90$ \\
\hline & pEGFP-N1-Ubc9 & $8 / 10$ & 40 to $>90$ \\
\hline
\end{tabular}

\section{Discussion}

As is well known, post-translational modifications play an important role in protein function through the regulation of their activity, turnover and localization and/or interactions. One such modification involves the covalent attachment of the small ubiquitin-related polypeptide SUMO (small ubiquitinlike modifier) to different cellular protein substrates $(8,18)$. Sumoylation has been implicated in the regulation of protein stability, protein-protein interactions, transcriptional activity and subcellular localization $(19,20)$. As an essential E2 

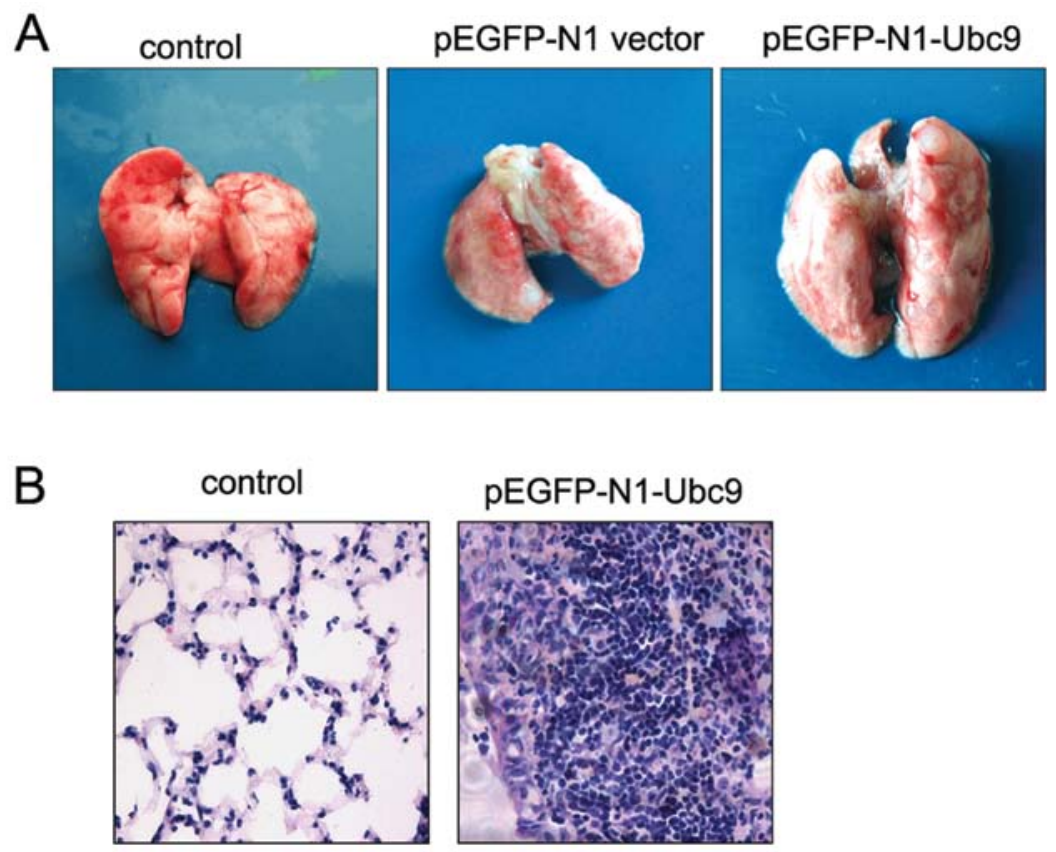

Figure 4. The overexpression of Ubc9 in NCI-H446 cells promotes lung cancer cell metastasis to lung. (A) Growth patterns of tumors formed by NCIH446+pEGFP-N1-vector cells or NCI-H446+pEGFP-N1-Ubc9 cells treated with intracardiac injection of tumor cells. (B) Histological analyses of lung metastatic tumors. Magnification, x100.

conjugating enzyme for sumoylation, Ubc9 plays a key role in sumoylation-mediated cellular pathways. Evidence suggests that Ubc9 is a tumor promoting factor. Ubc9 is well known for its key function in protein sumoylation and sumoylationregulated cellular pathways, ultimately affecting tumor initiation and progression (21). However, little is known about the regulation of Ubc9 in tumor cell invasion and metastasis. Our study provides evidence that Ubc9 promotes both lung cancer invasion and metastasis.

Ubc9 is the sole E2 conjugating enzyme required for protein sumoylation (22). Ubc9 plays a role in a number of important biological processes, such as progression through $M$ phase, DNA damage repair, maintenance of nuclear integrity and chromosome segregation. Several cell signaling molecules such as p53, Rad51, MITF, Smad4, c-Jun, Daxx, WT-1, NF- $\kappa \mathrm{B}$ and sex hormone receptors are target proteins of Ubc9 (23-26). In contrast to the ubiquitin pathway which utilizes several E2 conjugating enzymes, Ubc9 is the only known SUMO E2 enzyme and therefore a key regulator of the sumoylation pathway. As an essential E2 enzyme, Ubc9 is considered to play a central role in sumoylation-regulated cellular pathways which therefore suggests a putative role for sumo-regulation of pathways linked to tumorigenesis and drug resistance.

Ubc9 has been specifically linked to tumorigenesis. Ubc9 is overexpressed in several malignancies, such as lung adenocarcinoma (27), ovarian carcinoma (28), and melanoma (16). Antagonizing Ubc9 function in MCF-7 breast cancer cells transplanted in nude mice inhibited cell growth and increased apoptosis via Bcl-2 dependent mechanisms (29). These data suggest that Ubc9 may act by preventing activation of apoptotic pathways. In one study, ectopic expression of Ubc9 was shown to enhance the invasive ability of lung cancer cells. Additional studies have suggested that the Ubc9 gene may play a pivotal role in lung tumorigenesis and may potentially serve as a biomarker for both the diagnosis and prognosis of human lung cancer.

In accordance with previous studies $(16,30,31)$, higher levels of Ubc9 expression were detected in primary lung cancer as compared with normal lung tissue. Moreover, both protein and mRNA level of Ubc9 in NSCLC were higher than in SCLC. In patient tissue samples, the Ubc9 expression level was significantly correlated with more invasive lung cancer. These data suggest that $\mathrm{Ubc} 9$ is associated with the progression of lung cancer. Our study also provides experimental evidence that Ubc9 plays a crucial role in lung cancer cell invasion and migration. We transferred the eukaryotic expression construct pEGFP-Ubc9 to NCI-H446 cells and selected NCI-H446 single-clone cell lines stably overexpressing the Ubc9 gene. Using a Matrigel invasion assay, we found that the NCI-H446 cells overexpressing Ubc9 protein showed an enhanced invasion ability as compared to control cells. We also analyzed the effect of Ubc9 on cell migration and found similar results.

To further determine whether Ubc9 has a function in tumor metastasis, we performed in vivo metastasis assays by injecting transfected NCI-H446 cells into nude mice through the cardiac ventricle and measured metastasis to the lung. Similar to the results of our in vitro invasion assay, NCI-H446 cells overexpressing Ubc9 protein induced more metastatic nodules than the control cells. Moreover, the lung tumor nodules induced by the Ubc 9 overexpressing cells contained more foci than those induced by the control cells. The H\&E stain revealed that the tumor nodules induced by NCI-H446 cells overexpressing Ubc9 displayed the histomorphology of SCLC. These data indicate that NCI-H446 cells overexpressing Ubc9 protein have increased tumorigenicity which suggests that Ubc9 promotes lung cancer invasion and metastasis in vivo. Our study suggests that Ubc9 plays a causal 
role in cancer invasion and metastasis. This is likely due to the fact that Ubc9 is an essential enzyme for sumoylation and numerous important proteins, such as tumor suppressors or oncoproteins, are substrates for sumoylation. Similarly, Ubc9-mediated sumoylation has been shown to be involved in diverse cellular pathways (19). Therefore, cancer cells may have evolved mechanisms to target the basic functions of these protein modification pathways, involving microRNA regulation of Ubc9 at the post-transcriptional level, leading to its upregulation in tumors.

Metastasis is detected in $90 \%$ of lung cancer-related deaths (25). As metastasis involves different cellular processes than those that are involved in the onset and early stages of tumorigenesis, metastatic cancer can be more effectively treated if the metastasis process can be specifically targeted by therapeutics. Then, identifying key molecules involved in metastasis may aid substantially in the early diagnosis of metastatic lung cancer, thus reducing patient mortality. Our results indicate that Ubc9 may function as a potential molecular marker in metastatic lung cancer, and may be a new target for lung cancer therapy.

In summary, we provided clinical and experimental evidence that Ubc9 is differentially expressed in lung cancer cells and contributes to lung cancer cell migration and metastasis. Ubc9 may serve as a potential biomarker as a therapeutic target for cancer intervention.

\section{Acknowledgements}

The study was supported by Outstanding Scientific Fund of Shengjing Hospital.

\section{References}

1. Parkin DM, Bray F, Ferlay J and Pisani P: Global cancer statistics, 2002. CA Cancer J Clin 55: 74-108, 2005.

2. Jemal A, Siegel R, Ward E, Murray T, Xu J and Thun M: Cancer statistics, 2008. CA Cancer J Clin 58: 71-96, 2008.

3. Hirsch FR, Franklin WA, Gazdar AF and Bunn PA Jr: Early detection of lung cancer: clinical perspectives of recent advances in biology and radiology. Clin Cancer Res 7: 5-22, 2001.

4. Erridge S, Moller H, Price A and Brewster D: International comparisons of survival from lung cancer: pitfalls and warnings. Nat Clin Pract Oncol 4: 570-577, 2007.

5. Kligerman $\mathrm{S}$ and White $\mathrm{C}$ : Epidemiology of lung cancer in women: risk factors, survival, and screening. AJR Am J Roentgenol 196: 287-295, 2011

6. Cagle PT, Allen TC, Dacic S, Beasley MB, Borczuk AC, Chirieac LR, Laucirica R, Ro JY and Kerr KM: Revolution in lung cancer: new challenges for the surgical pathologist. Arch Pathol Lab Med 135: 110-116, 2011.

7. Steeg PS: Metastasis suppressors alter the signal transduction of cancer cells. Nat Rev Cancer 3: 55-63, 2003.

8. Muller S, Hoege C, Pyrowolakis G and Jentsch S: SUMO, ubiquitin's mysterious cousin. Nat Rev Mol Cell Biol 2: 202-210, 2001.
9. Gill G: SUMO and ubiquitin in the nucleus: different functions, similar mechanisms? Genes Dev 18: 2046-2059, 2004.

10. Hay RT: SUMO: a history of modification. Mol Cell 18: 1-12, 2005.

11. Lin D, Tatham MH, Yu B, Kim S, Hay RT and Chen Y: Identification of a substrate recognition site on Ubc9. J Biol Chem 277: 21740-21748, 2002.

12. Duan X, Trent JO and Ye H: Targeting the SUMO E2 conjugating enzyme Ubc9 interaction for anti-cancer drug design. Anticancer Agents Med Chem 9: 51-54, 2009.

13. Niedenthal R: Ubc9 fusion-directed SUMOylation (UFDS). Biochem Soc Trans 35: 1430-1432, 2007.

14. Galanty Y, Belotserkovskaya R, Coates J, Polo S, Miller KM and Jackson SP: Mammalian SUMO E3-ligases PIAS1 and PIAS4 promote responses to DNA double-strand breaks. Nature 462: 935-939, 2009.

15. Vertegaal AC: SUMO chains: polymeric signals. Biochem Soc Trans 38: 46-49, 2010.

16. Ahn JH, Xu Y, Jang WJ, Matunis MJ and Hayward GS: Evaluation of interactions of human cytomegalovirus immediate-early IE2 regulatory protein with small ubiquitin-like modifiers and their conjugation enzyme Ubc9. J Virol 75: 3859-3872, 2001.

17. Moschos SJ, Smith AP, Mandic M, et al: SAGE and antibody array analysis of melanoma-infiltrated lymph nodes: identification of Ubc9 as an important molecule in advanced-stage melanomas. Oncogene 26: 4216-4225, 2007.

18. Wu F, Zhu S, Ding Y, Beck WT and Mo YY: MicroRNAmediated regulation of Ubc9 expression in cancer cells. Clin Cancer Res 15: 1550-1557, 2009.

19. Johnson ES: Protein modification by SUMO. Annu Rev Biochem 73: 355-382, 2004

20. Geiss-Friedlander R and Melchior F: Concepts in sumoylation: a decade on. Nat Rev Mol Cell Biol 8: 947-956, 2007.

21. Driscoll JJ, Pelluru D, Lefkimmiatis K, et al: The sumoylation pathway is dysregulated in multiple myeloma and is associated with adverse patient outcome. Blood 115: 2827-2834, 2010.

22. Moschos SJ, Jukic DM, Athanassiou C, et al: Expression analysis of Ubc9, the single small ubiquitin-like modifier (SUMO) E2 conjugating enzyme, in normal and malignant tissues. Hum Pathol 41: 1286-1298, 2010.

23. Vertegaal AC: Small ubiquitin-related modifiers in chains. Biochem Soc Trans 35: 1422-1423, 2007.

24. Wu SY and Chiang CM: p53 sumoylation: mechanistic insights from reconstitution studies. Epigenetics 4: 445-451, 2009.

25. Nowak $M$ and Hammerschmidt M: Ubc9 regulates mitosis and cell survival during zebrafish development. Mol Biol Cell 17: 5324-5336, 2006.

26. Fu M, Wang C, Wang J, Zhang X, et al: Androgen receptor acetylation governs transactivation and MEKK1-induced apoptosis without affecting in vitro sumoylation and trans-repression function. Mol Cell Biol 22: 3373-3388, 2002.

27. Vatsyayan J, Qing G, Xiao G and Hu J: SUMO1 modification of NF-kappaB2/p100 is essential for stimuli-induced p100 phosphorylation and processing. EMBO Rep 9: 885-890, 2008.

28. Moschos SJ and Mo YY: Role of SUMO/Ubc9 in DNA damage repair and tumorigenesis. J Mol Histol 37: 309-319, 2006.

29. Mo YY and Moschos SJ: Targeting Ubc9 for cancer therapy. Expert Opin Ther Targets 9: 1203-1216, 2005.

30. Lu Z, Wu H and Mo YY: Regulation of bcl-2 expression by Ubc9. Exp Cell Res 312: 1865-1875, 2006.

31. Mo YY, Yu Y, Theodosiou E, Ee PL and Beck WT: A role for Ubc9 in tumorigenesis. Oncogene 24: 2677-2683, 2005. 